\title{
5 Qualitative and quasi-quantitative monitoring of drinking water through the use of a smart electronic tongue
}

10

Alvaro A. Arrieta ${ }^{1}$, Said Marquez ${ }^{1}$, Jorge Mendoza ${ }^{2}$

15

${ }^{1}$ Department of Biology and Chemistry, University of Sucre, Sincelejo, postal code 700001, Colombia.

${ }^{2}$ Department of Mechanical Engineering, University of Cordoba, Monteria, postal code 230002, Colombia.

Correspondence to: Alvaro A. Arrieta (alvaro.arrieta@unisucre.edu.co) 
https://doi.org/10.5194/dwes-2021-12

Preprint. Discussion started: 14 January 2022

(c) Author(s) 2022. CC BY 4.0 License.

(c) (i)

Abstract. Monitoring the quality of drinking water is undoubtedly an issue of global concern. In this sense, the new analytical approaches that incorporate new technologies are without doubt relevant. The aim of this work is to present the evaluation of a smart electronic tongue device as an alternative for qualitative and quantitative monitoring of drinking water. For this, a smart electronic tongue used consisted of cross-sensitive polypyrrole sensor array, coupled to a multi-channel electronic system (multipotentiostat) based on PSoC technology controlled from a smartphone with a data acquisition and control app. This device was used in the monitoring of drinking water from the Sincelejo city aqueduct system and water samples collected and analyzed by the public health agency were used. The voltammetric measurements carried out with the smart electronic tongue showed cross-sensitivity of the polypyrrole sensor array, which allowed the discrimination of the samples through analysis of principal components by artificial neural networks. In addition, the records made with the smart electronic tongue allowed, through partial least square by artificial neural networks estimating the concentrations of some important analytes in the evaluation of the physicochemical quality of drinking water with $\mathrm{R} 2$ values higher than 0.70 . The results allowed to conclude that the smart electronic tongue constitutes a valuable analytical tool that allows, in a single measure, to perform qualitative and quantitative chemical analysis, it is also a fast, portable method that can complement traditional analyzes.

\section{Introduction}

50 In recent decades, interest in the quality control of food, drinking water, beverages and in general of products for human consumption. To perform this control, in addition to reliable methods, it is sought to have fast methods that allow real-time and online surveillance. In the particular case of drinking water, analyses are carried out using techniques and methods that mostly require sophisticated and specialized equipment, such as UV-Vis spectrometers, chromatographs, mass spectrometers, infrared spectrometers, atomic absorption spectrometers, among others (Richardson et al., 2017; Rice et al., 2017). In general,

55 these equipment are expensive and require qualified personnel for their operation, they are also bulky equipment that consume significant amounts of energy and can only operate in facilities or laboratories suitable for their operation. Further, most of the analyses require sample pre-treatment, long processing times and generate a considerable amount of chemical waste. These conditions and restrictions in traditional analytical approaches, have led to search new technological alternatives cheaper, faster, easier, efficient and effective. The above has led to the generation of new technologies, among which the electronic 60 tongues.

Electronic tongues are analytical devices, made up of a non-specific chemical sensor array, with cross-sensitivity, coupled to a multichannel measurement system and an app or software that allows pattern recognition (Vlasov et al., 2005). A certain 
https://doi.org/10.5194/dwes-2021-12

Preprint. Discussion started: 14 January 2022

(c) Author(s) 2022. CC BY 4.0 License.

(c) (i)

analogy can be established between the human gustatory system and electronic tongues, in such a way that we can find some approximations in its structure and principles of operation. In Figure 1 can be seen a comparative scheme that shows the analogies between the functioning of the human taste system and the artificial system.

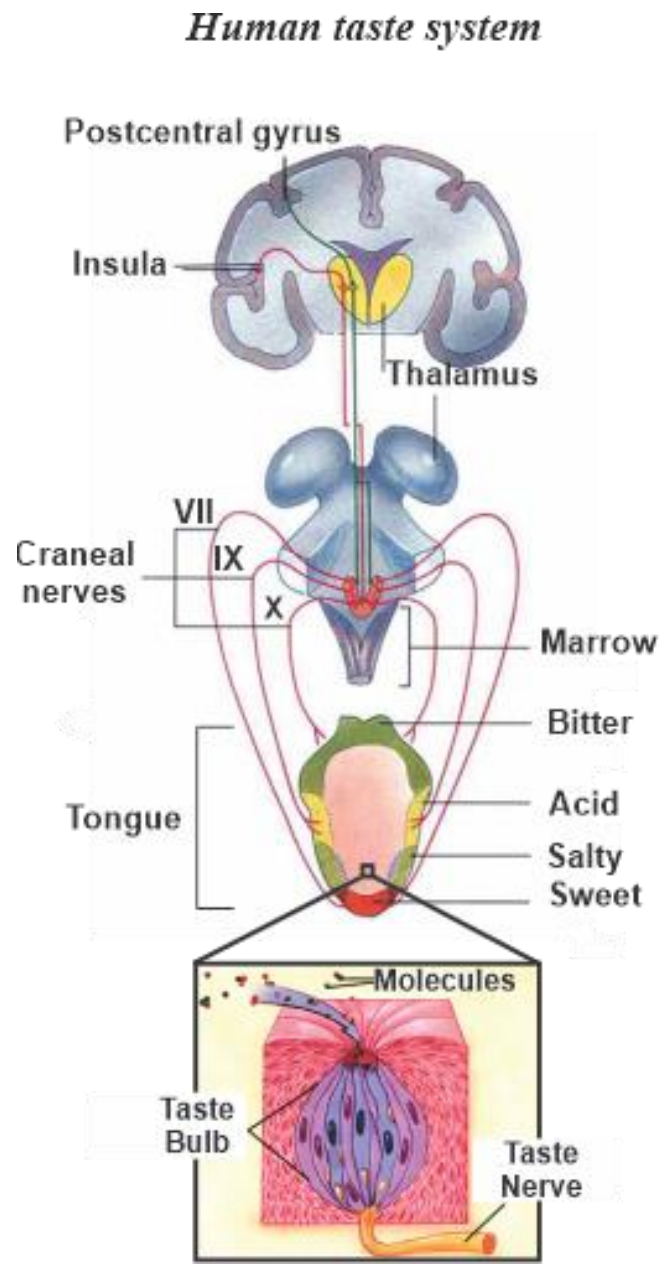

\section{Smart electronic tongue}

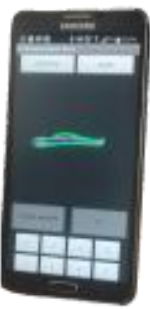

Data treatment

(Artificial neural networks)

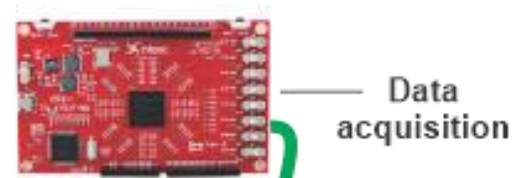

Figure 1. Functional analogy between the human taste system and an artificial system (smart electronic tongue).

70 Although in the electronic tongue devices have been used various analytical principles such as optical, mass, frequency measurements, among others (Khan et al., 2016; li et al., 2019; Kovacs et al., 2020; Sehra et al., 2004; Aydemir and Ebeoglu, 2018). Devices based on potentiometric and voltammetric electrochemical measurements have been more widely accepted and have shown their effectiveness in the analysis of different types of beverages (Arrieta et al., 2019; Belugina et al., 2020; Totova and Nachev, 2020; Marx et al., 2017). However, electronic tongues based on voltammetric measurements have some advantages such as greater ease of processing the sensors, low sensitivity to electronic noise, high analytical sensitivity and versatility in terms of the voltammetric technique used (square wave, cyclic, pulse, etc.). 
https://doi.org/10.5194/dwes-2021-12

Preprint. Discussion started: 14 January 2022

(c) Author(s) 2022. CC BY 4.0 License.

(c) (i)

The electronic tongues have been used in the analysis of mineral waters (Sipos et al., 2012), waste waters (Legin et al., 2019) and bottled waters (Dias et al., 2015) and qualitative (sample classification) and quantitative analyses on analytes such as $\mathrm{Na}^{+}$, $\mathrm{K}^{+}, \mathrm{Ca}^{2+}, \mathrm{Cl}^{-}, \mathrm{NaCl}, \mathrm{NaN}_{3}, \mathrm{NaHSO}_{3}$, ascorbic acid and $\mathrm{NaOC}$ (Winquist et al., 2011; Atas et al., 2020), among others. However,

80 no reports have been found on the application of this technology in the analysis of drinking water from household networks and on the analytes of greatest interest in the evaluation of its physicochemical quality such as hardness, alkalinity, chlorides, sulphates, chlorine, etc.

The electronic tongue devices reported, are mostly laboratory equipment, which limits their portability for on-site analysis. In this work, the application of a portable smart electronic tongue is reported, made up of miniaturized polypyrrole sensor array,

85 a multichannel device made under PSoC technology (microchip technology) and a smartphone equipped with an Android app. The recorded data were analyzed with methods of pattern recognition and regression by partial least squares (PLS) based on artificial neural networks. This smart electronic tongue was used to qualitatively and quantitatively analyze samples taken from the 22 points (hydrants) of the distribution system. The smart electronic tongue due to its characteristics, allows its complete portability and in-site and online analysis, which represents a significant advance in the monitoring and control of water quality since it can allow quick and corrective responses. early to avoid possible damage to the health of populations.

\section{Materials and Methods}

\subsection{Collection of samples and sampling area}

The samples were taken in the supply networks at the hydrants defined by the drinking water service provider company (ADESA SAESP), located in communes 1, 2, 3, 4, 5, 6, 7 and 9 of the city of Sincelejo - Colombia (Sincelejo mayorship,

95 2017), located in the northeast of the country at $9^{\circ} 18$ " north latitude, $75^{\circ} 23^{\prime \prime}$ latitude, west of the Greenwich meridian, altitude of 213. For the sampling, the national guidelines on the minimum number of samples and the distribution of sampling points established for the populations according to their number of inhabitants were taken into account.

The sampling hydrants were defined taking into account the programming of the operating company of the aqueduct system and the entity of surveillance and control of the quality of drinking water. Table 1 presents the summary of the programming of the sampling carried out, in which the location or geographical area was noted; commune (C) and sector (S) and the place of sampling point or hydrant $(\mathrm{H})$. For the sampling procedure, the protocols established by the national health authority were followed (National Institute of Health, 2019).

The samples were divided into aliquots to carry out the different analyses. The characterization of the physicochemical parameters was carried out in the facilities of the departmental reference laboratory of Public Health of the Department of Health of Sucre, an entity in charge of exercising control and monitoring of water for human consumption and its characteristics. The methods and techniques used for each of the parameters analyzed were those established in the standard analysis methods required by national regulations (Richardson et al., 2017; Rice et al., 2017). 
Table 1. Drinking water sampling location data.

\begin{tabular}{|c|c|c|}
\hline \multirow{2}{*}{$\begin{array}{l}\text { Sample } \\
\text { code }\end{array}$} & Location & $\begin{array}{c}\text { Sampling } \\
\text { location }\end{array}$ \\
\hline & $\begin{array}{c}\text { (Commune and } \\
\text { sector) }\end{array}$ & (Hydrant) \\
\hline M1 & $\mathrm{C} 1 \mathrm{~S} 3$ & H2014 \\
\hline M2 & $\mathrm{C} 2 \mathrm{~S} 18$ & H2016 \\
\hline M3 & $\mathrm{C} 3 \mathrm{~S} 7$ & H2015 \\
\hline M4 & $\mathrm{C} 3 \mathrm{~S} 8$ & H2013 \\
\hline M5 & C3S8 & H2012 \\
\hline M6 & $\mathrm{C} 4 \mathrm{~S} 12$ & H2011 \\
\hline M7 & $\mathrm{C} 4 \mathrm{~S} 12$ & H2008 \\
\hline M8 & $\mathrm{C} 4 \mathrm{~S} 15$ & H2029 \\
\hline M9 & $\mathrm{C} 4 \mathrm{~S} 15$ & H2007 \\
\hline M10 & $\mathrm{C} 5 \mathrm{~S} 25$ & H2030 \\
\hline M11 & C5S26 & H2027 \\
\hline M12 & C5S33 & H2004 \\
\hline M13 & C5S33 & H2005 \\
\hline M14 & C5S34 & H2028 \\
\hline M15 & $\mathrm{C} 6 \mathrm{~S} 23$ & H2019 \\
\hline M16 & C6S23 & H2017 \\
\hline M17 & C7S27 & H2003 \\
\hline M18 & C7S34 & H2026 \\
\hline M19 & C7S49 & H2001 \\
\hline M20 & C7S51 & H2006 \\
\hline M21 & C9S40 & H 2022 \\
\hline M22 & C9S40 & H2024 \\
\hline
\end{tabular}

\section{2 smart electronic tongue device and measurements}

The smart electronic tongue developed in our laboratory consisted of a polypyrrole voltammetric sensor array a portable multipotentiostat controlled from a smartphone. For the elaboration of the sensor array, a card with screen-printed electrodes from BVT Technologies (AC9C) was used, which consists of an auxiliary or counter electrode (CE), an $\mathrm{Ag} / \mathrm{AgCl}$ reference 
https://doi.org/10.5194/dwes-2021-12

Preprint. Discussion started: 14 January 2022

(c) Author(s) 2022. CC BY 4.0 License.

(c) (i)

115 electrode (ER) and seven working electrodes of graphite, which were used as substrates for the generation of the sensors. Thus, the sensor array consisted of seven polypyrrole (PPy) voltammetric sensors doped with seven different doping agents: PPy / DBS (PPy doped with sodium dodecyl benzene sulfonate), PPy / SO4 (PPy doped with sodium sulphate), PPy / SF (PPy doped with sodium persulfate), PPy / FCN (PPy doped with sodium ferrocyanide), PPy / TSA (PPy doped with p-toluene sulfonic acid), PPy / AQDS (PPy doped with disodium salt of the acid anthraquinone-2,6-disulfonic) and PPy / PC (PPy doped with lithium perchlorate).

The sensor array was prepared by chronoamperometric electropolymerization of pyrrole at $0.8 \mathrm{~V}$, using an EG\&G 2273 PAR potentiostat/galvanostat, controlled with PowerSuite software. The PPy with each of the dopants was electrodeposited on the graphite substrates arranged in a circular way on the commercial AC9C card. Table 2 shows the experimental conditions used in the synthesis of the sensor array.

Table 2. Experimental conditions for electropolymerization of the sensor array

\begin{tabular}{llcc}
\hline Sensor & Acronym & $\begin{array}{c}\text { Concentration } \\
\text { Pyrrole/Doping Agent [M] }\end{array}$ & $\begin{array}{c}\text { Polymerization } \\
\text { time (s) }\end{array}$ \\
\hline S1 & PPy/SO4 & $0.1 / 0.05$ & 55 \\
\hline S2 & PPy/DBS & $0.1 / 0.1$ & 50 \\
\hline S3 & PPy/SF & $0.1 / 0.05$ & 65 \\
\hline S4 & PPy/FCN & $0.1 / 0.1$ & 60 \\
\hline S5 & PPy/PC & $0.1 / 0.1$ & 60 \\
\hline S6 & PPy/TSA & $0.1 / 0.1$ & 70 \\
\hline S7 & PPy/ AQDS & $0.1 / 0.05$ & 60 \\
\hline \hline
\end{tabular}

The portable multipotentiostat was made on a FREESOC card with a PSoC 5LP microchip (Programmable System on Chip), which was programmed with the $\mathrm{PSoC}$ creator software. This electronic device was designed to simultaneously register the voltammetric signals of the seven sensors of the array through seven measurement channels. In addition, a Bluetooth card was incorporated for data transmission to a smartphone equipped with an Android app designed to control the device and record data. Details on the electrochemical polymerization techniques, the development of the electronic device and the control Android app have been previously reported (Arrieta and Fuentes, 2016; Arrieta et al., 2015, Arrieta et al., 2016). Figure 2 presents an image of the smart electronic tongue and its three fundamental components are highlighted.

135 The measurements carried out with the smart electronic tongue were carried out on $10 \mathrm{~mL}$ of sample at room temperature and without previous treatment. 7 replicates of each measure were made. The voltammetric signals were recorded at a sweep rate of $100 \mathrm{mV} \mathrm{s}^{-1}$, in a potential range of $-1.0 \mathrm{~V}$ to $0.5 \mathrm{~V}$ with an initial potential of $0.0 \mathrm{~V}$. 
https://doi.org/10.5194/dwes-2021-12

Preprint. Discussion started: 14 January 2022

(c) Author(s) 2022. CC BY 4.0 License.

(c) (i)

\subsection{Data processing and evaluation of the qualitative and quantitative analysis carried out with the smart electronic tongue}

140 From the signals obtained during the measurements carried out with the smart electronic tongue, the current data generated by the sensor array was recorded. Each sensor generated a voltammogram of each sample, composed of 100 data, which allowed having 700 data with the entire sensor array, each one of them was a variable in the data matrix for each sample, which constituted a species "fingerprint" of the sample. Thus, when analyzing all the samples, a matrix of 107,800 data was constructed (700 variables x 22 samples x 7 replicates).

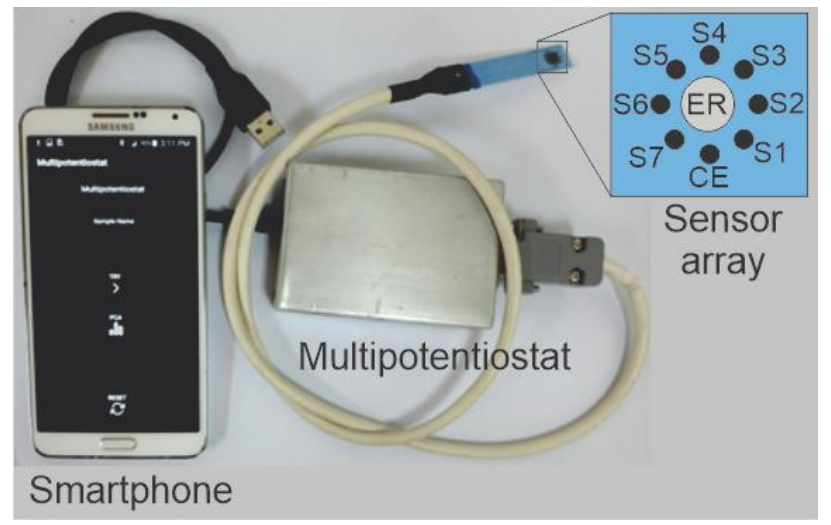

Figure 2. Image of smart electronic tongue formed by the miniaturized sensor array, portable electronic device and smartphone with an Android app.

150 To evaluate the classification capacity (qualitative analysis) in drinking water samples, the matrix was subjected to a pattern recognition analysis by applying artificial neural networks for principal component analysis. To validate the results and evaluate the reproducibility of the method, the measurement procedure was repeated on a group different of samples, sampled 15 days after the first discrimination test and it was applied the same sampling protocol, measurement with the smart electronic tongue and treatment of data.

155 On the other hand, a quantitative analysis was carried out from regression models using artificial neural networks for partial least squares, to establish a correlation between the voltammetric measurements registered with the smart electronic tongue and the concentrations of eight of the most important physicochemical parameters in evolution of the quality of drinking water (alkalinity, calcium, residual chlorine, chlorides, total hardness, phosphates, magnesium and sulphates). The physicochemical parameters were determined using the traditional methods validated by the norms and standardized methods (Richardson et

160 al., 2017; Rice et al., 2017). That is, the prediction models created were generated from the data obtained in the characterization process with the smart electronic tongue (matrix X, independent variables) and the physicochemical parameters determined using the traditional methods in each water sample ( $Y$ matrix, dependent variables). In this way, the concentrations of the 
https://doi.org/10.5194/dwes-2021-12

Preprint. Discussion started: 14 January 2022

(c) Author(s) 2022. CC BY 4.0 License.

(c) (i)

physicochemical parameters of drinking water determined by traditional methods were evaluated against those predicted by smart electronic tongue through regression models.

165 The chemometric treatment of the data was carried out using specific artificial neural networks designed under the MATLAB V 7.12 program using Neural Network Toolbox v.3.0 (Kong et al., 2017). The data were not pretreated and to select the number of latent variables, a "cross-validation" was performed before building the prediction model.

\section{Results and discussions}

\subsection{Voltammetric Response of Smart Electronic Tongue}

170 Once the samples were collected at the sampling points, the respective measurements were made by using the smart electronic tongue in an aliquot of $10 \mathrm{~mL}$. Each measurement carried out with the smart electronic tongue lasted approximately 4 minutes per sample, which represents a total of about 88 minutes in the 22 samples. The signals were recorded on the smartphone using the Android app designed for this purpose. The voltammetric signals showed cross sensitivity in the sensors. that is, each sensor presented a particular response in the same sample, which means that each one provides information about the analyzed sample, which constitutes the "fingerprint". In Figure 3, the response of the sensor array against sample M1 (C1S3-H2014) is presented as an example. It can be seen in the graphs, that the voltammetric signal of the sensor S1 (PPy/SO4), shows an anodic process at $-0.249 \mathrm{~V}$ and in the cathodic sweep a reduction process can be observed at $-0.875 \mathrm{~V}$. The response of the sensor S2 (PPy/DBS) shows a redox process, with an oxidation peak at - $0.109 \mathrm{~V}$ and a wide reduction peak in the cathodic scan at $-0.799 \mathrm{~V}$.

180 The signal recorded with S3 sensor (PPy/SF) consists of two anodic processes at $0.249 \mathrm{~V}$ and $-0.351 \mathrm{~V}$. On the other hand, the voltammetric response of $\mathrm{S} 4$ sensor $(\mathrm{PPy} / \mathrm{FCN})$ presents a signal with poorly defined anodic and cathodic process at 0.287 $\mathrm{V}$ and $-0.124 \mathrm{~V}$, respectively. The voltammetric responses of the S5 sensor (PPy/PC) and S6 (PPy/TSA) show in both cases a redox process, composed of an anodic peak at $0.03 \mathrm{~V}$ for PPy/PC and - $0.252 \mathrm{~V}$ for PPy/TSA, and in cathodic scanning it can be seen that PPy/PC presents the reduction peak at $-0.747 \mathrm{~V}$, on the other hand, the PPy/TSA cathodic scanning shows the reduction peak at - $0.821 \mathrm{~V}$. The voltammetric signal of the S7 sensor (PPy / AQDS), presented an oxidation process in the cathodic wave at $0.202 \mathrm{~V}$.

Besides, the cross sensitivity was evaluated, the capacity of the sensor array to generate particular signals in front of each one of the samples, which allows to have a fingerprint of each one of them. In Figure 4 is shown as example, the behavior of S1 sensor (PPy/SO4) against some water samples taken at different sampling points (M1, M2, M3, M4 and M5). As can be seen

190 in Figure 4, the signals recorded in the water samples are remarkably different. Thus, the main differences are observed in the position of the peaks (redox potentials) of each of the sensors and the shapes of the curve. This allows obtaining information from the analyzed water samples. Starting from this fact, and to extract the information contained in the signals, a pattern recognition analysis was performed using artificial neural networks for principal component analysis. 
https://doi.org/10.5194/dwes-2021-12

Preprint. Discussion started: 14 January 2022

(c) Author(s) 2022. CC BY 4.0 License.

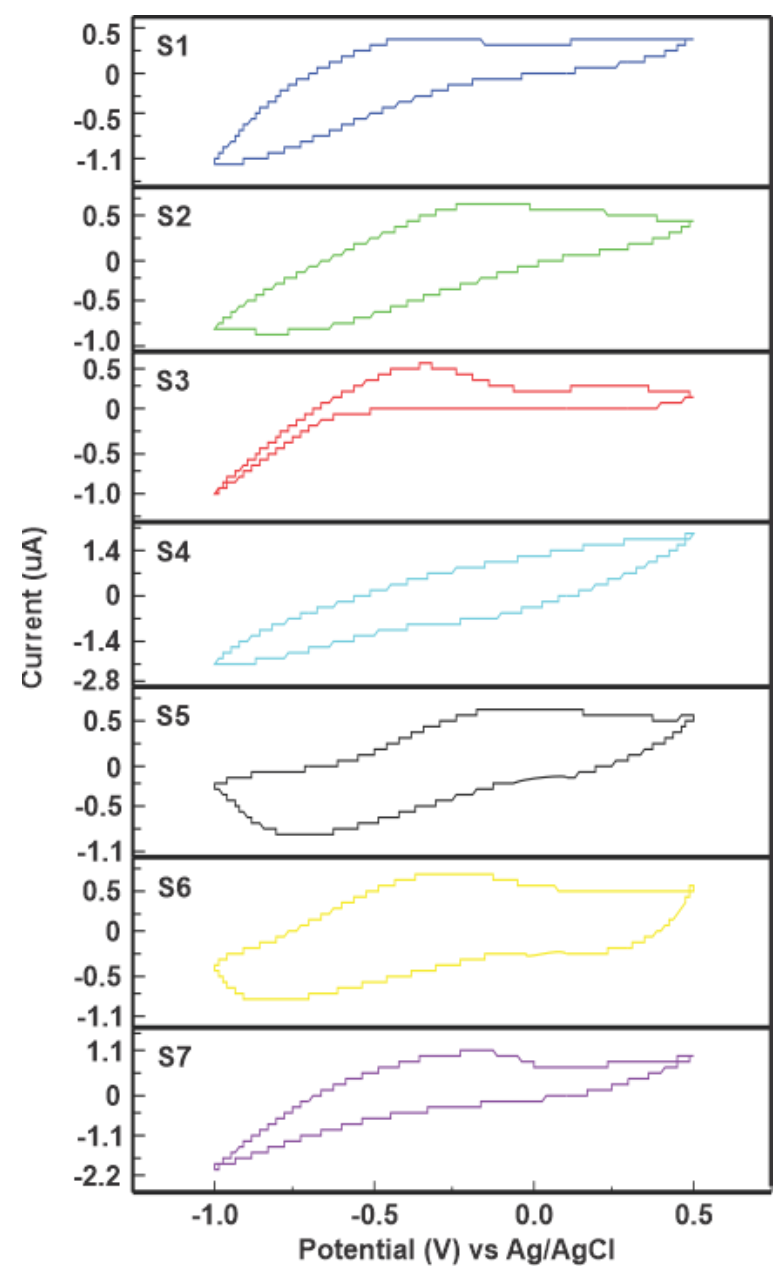

Figure 3. Voltammetric signals from the smart electronic tongue sensor array recorded in the drinking water sample M1

(C1S3 - H2014)

In summary, it could be shown that the shape and position (redox potentials) of the peaks in the voltammetric signals are markedly different in each of the sensors and a different signal pattern was recorded in each sample, allowing to have together a "fingerprint" of each one. This fact is important because the variety of responses obtained allowed us to obtain the necessary information to be able to analyze qualitatively and quantitatively the different water samples. In general terms, the signals are related to the entry and exit of ionic species from the water samples in the polymeric film of the polypyrrole (sensor) to maintain its electroneutrality, which is why the signals obtained contain information of each of the samples analyzed. 


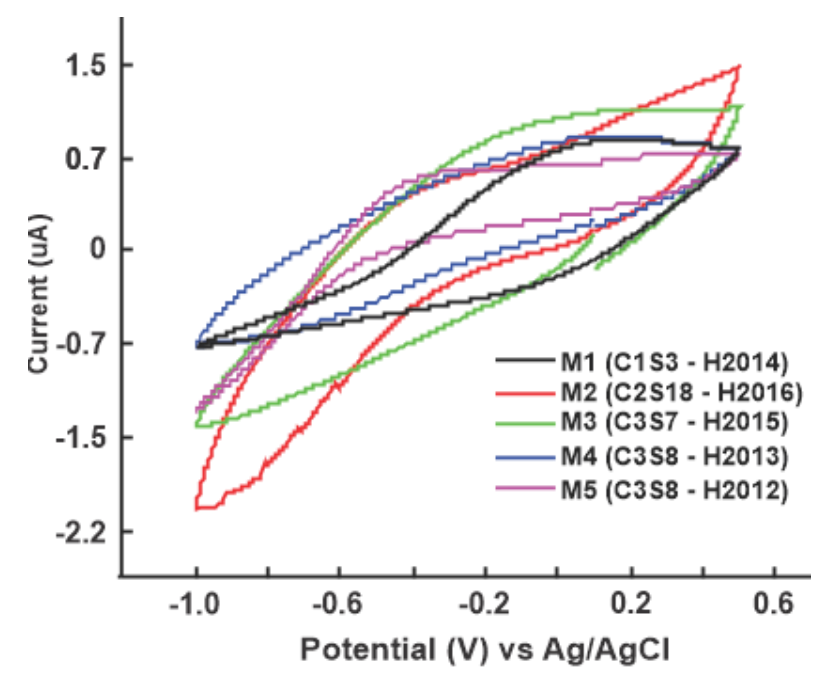

Figure 4. Voltammetric signals of the S1 sensor (PPy/SO4) recorded in different samples of drinking water.

\subsection{Qualitative analysis}

From the recorded signals, a matrix was constructed with the values obtained in each of the measurements. The matrix was used to perform a pattern recognition analysis to classify the samples, this procedure was performed by applying artificial neural networks for principal component analysis, a fact that allowed the extraction of the greatest amount of information due to the non-linearity of the method. Figure 5 shows the result obtained from the pattern recognition analysis by artificial neural networks for principal components, applied to the values supplied by the voltammetric signals recorded for the different water samples. The two principal components represented show a variance of $72.09 \%$.

In Figure 5, each point corresponds to a sample taken from a hydrant or sampling point taken in the respective geographical area (commune $\mathrm{C}$, sector $\mathrm{S}$ and hydrant $\mathrm{H}$ ). The samples maintain the nomenclature defined in table 1 . Thus, for example, sample M1 in table 1 corresponds to the sample taken at C1S3 and sampling point or hydrant H2014. The first principal component (PC 1) summarizes the most information with 56.49\% and the second principal component (PC 2) also collects a significant amount of $15.85 \%$. As can be seen, the different samples analyzed are remarkably distributed in the plane of the principal components with a higher concentration close to zero in both axes. In the area located in the lower right part of the graph (Figure 5 a), groups of samples appear that due to the high concentration of points (samples) may appear to be overlapping. However, when enlarging the area, it can be seen that none of the samples overlap (Figure $5 \mathrm{~b}$ ).

The samples with the greatest separation M1 (C1S3-H2014), M2 (C2S18-H2016), M3 (C3S7-H2015), M4 (C3S8-H2013) and

225 M5 (C3S8-H2012), belong to communes 1, 2 and 3, which are found in the western part of the city, with sample M1 the one with the highest degree of separation and the only sample from commune 1, samples M4 and M5 that belong to the same commune and the same sector present a certain proximity. This trend in the spatial distribution in the samples, without forming defined groups, is possibly due to the fact that as the water supply is made from the treatment site (re-pumping) towards the geographical location areas of the hydrants, their physicochemical characteristics vary slightly during its journey, possibly due 
https://doi.org/10.5194/dwes-2021-12

Preprint. Discussion started: 14 January 2022

(c) Author(s) 2022. CC BY 4.0 License.

(c) (i)

230 to factors such as temperature, time and date of sampling, aeration processes, maintenance and cleaning of networks, among others. This result showed a good discrimination capacity of the smart electronic tongue against drinking water samples, which evidence that the information registered by the sensor array is useful, because they capture the particularities of each sample allowing to carry out your discrimination.

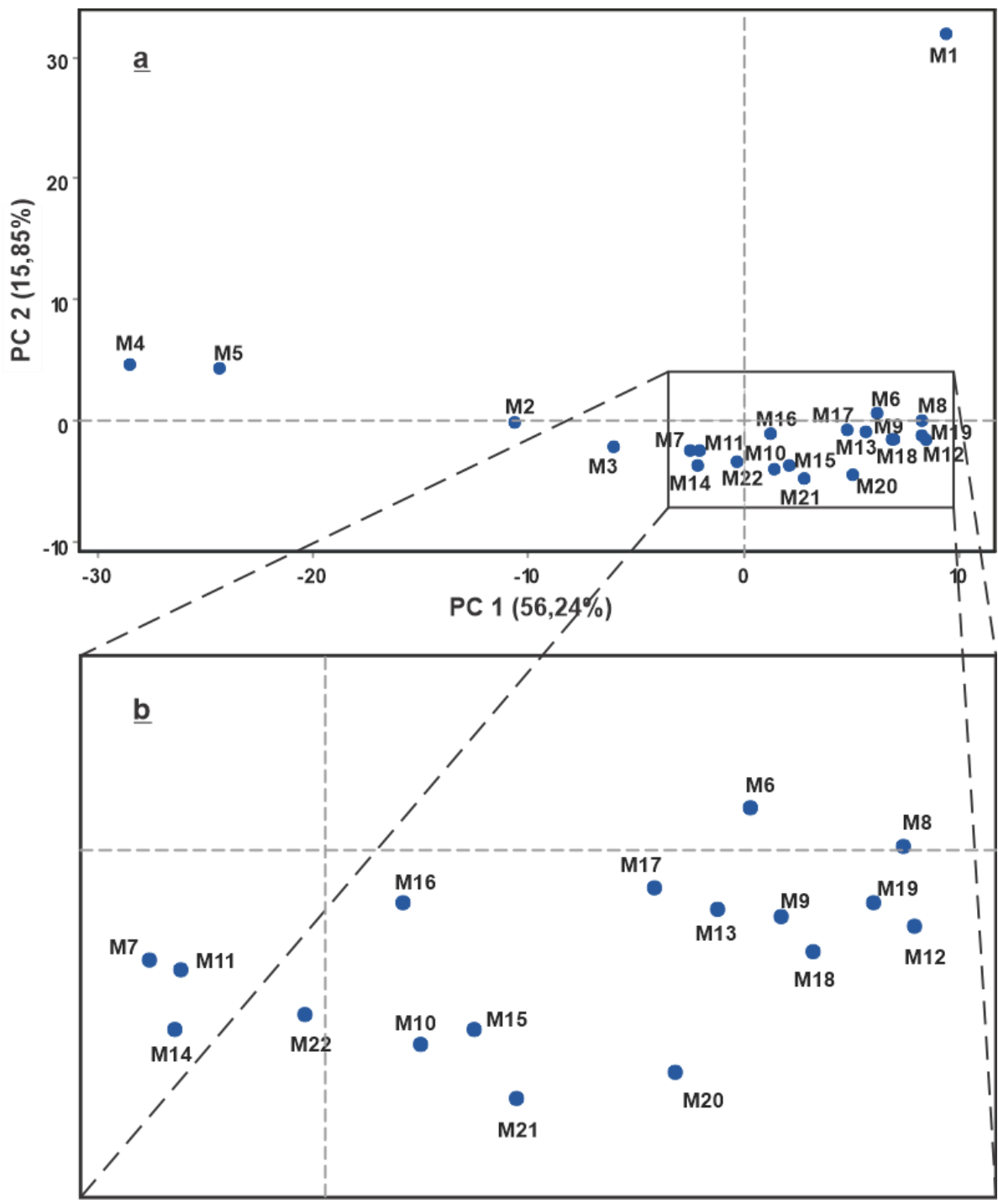

Figure 5. Plot of principal component score of signals collected in drinking water samples by smart electronic tongue.

Furthermore, a second test was carried out to corroborate the quality and reproducibility of these results. This trial consisted of repeating the experiences after 15 days. For this, a new group of samples collected at the same points was used and following the same protocols for sampling and recording signals with the smart electronic tongue. In this way, after treating the data with 
https://doi.org/10.5194/dwes-2021-12

Preprint. Discussion started: 14 January 2022

(c) Author(s) 2022. CC BY 4.0 License.

(c) (i)

the artificial neural network method for principal component analysis, a new principal component scores graph was generated from the new experiments.

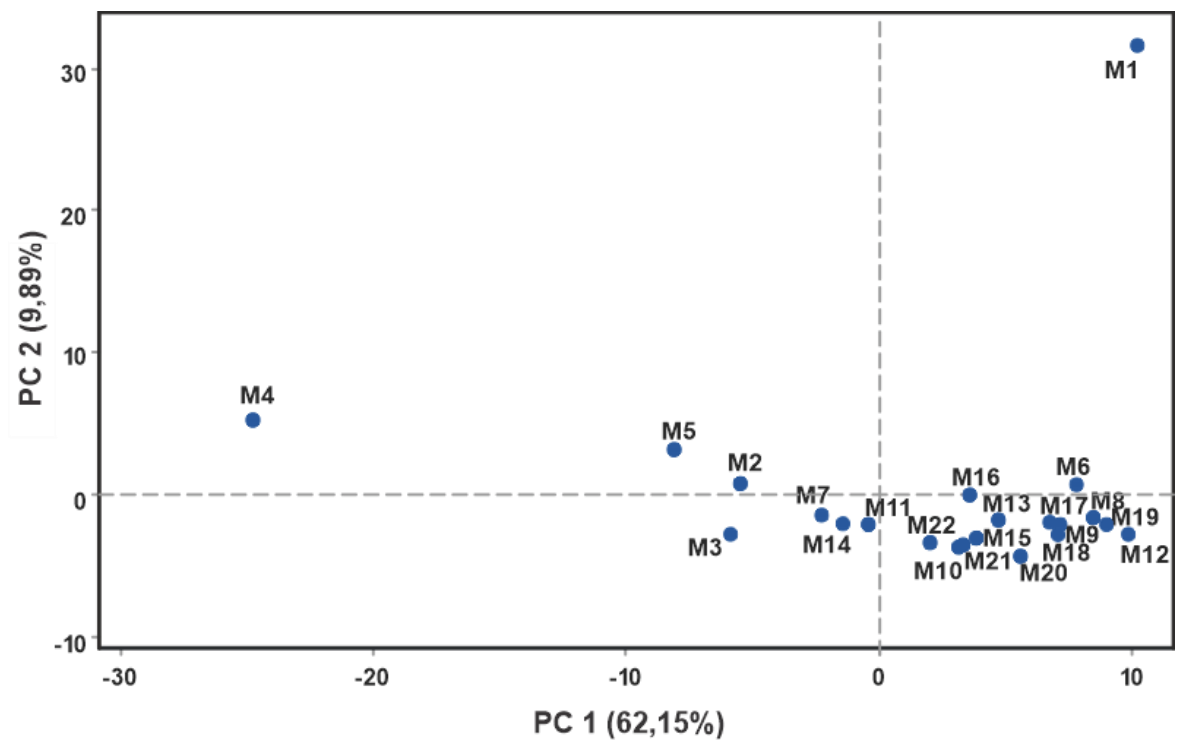

Figure 6. Plot of principal component scores of replicated tests after 15 days in a new group of drinking water samples measured with the smart electronic tongue.

Figure 6 shows the graph of principal component scores obtained with the second test. When comparing the distribution and the positions of the samples with those obtained from the experiments carried out in the first test (Figure 5), it can be observed a great similarity in the results. The information collected for PC 1 and PC 2 was $62.15 \%$ and $9.89 \%$ respectively, for a total of $72.04 \%$ of the information collected for the total variance, a value very similar to that obtained in the first trial (72.09\%). Although there are small variations, which may be the product of differences in the physicochemical characteristics of the samples, there is a high degree of reproducibility. Therefore, the results obtained with the analysis of the 22 samples and their replicas, showed an excellent discrimination capacity of the smart electronic tongue, with reproducible discrimination results.

\subsection{Quasi-quantitative Analysis}

One of the most interesting aspects of electronic tongues is the fact that in addition to allowing classifications of samples to evaluate their behavior in terms of global characteristics, it can also provide quantitative or quasi-quantitative information about the samples. In such a way that offers information on their content or concentration of chemical components at a quantitative level. In this sense, the ability of the smart electronic tongue to provide quantitative information of the water samples under study was explored, by obtaining correlations between the voltammetric measurements recorded by the smart 
https://doi.org/10.5194/dwes-2021-12

Preprint. Discussion started: 14 January 2022

(c) Author(s) 2022. CC BY 4.0 License.

(c) (i)

electronic tongue and the concentration of some compounds or substances present in drinking water samples. For this, the data of the two sets of 22 samples were taken to guarantee the robustness of the resulting models.

To carry out the extraction of quantitative information, regression models of artificial neural networks for partial least squares were used and eight relevant physicochemical parameters were chosen in the evaluation of the quality of drinking water (hardness, alkalinity, chlorides, residual chlorine, sulphates, magnesium, calcium and phosphates).

The results of the application of the regression analysis are shown in Figures 7 and 8 (the results were divided into 2 figures to improve the visualization). In figure 7, the regression graphs obtained from the application of the models on the parameters alkalinity, calcium, hardness and phosphates are presented.
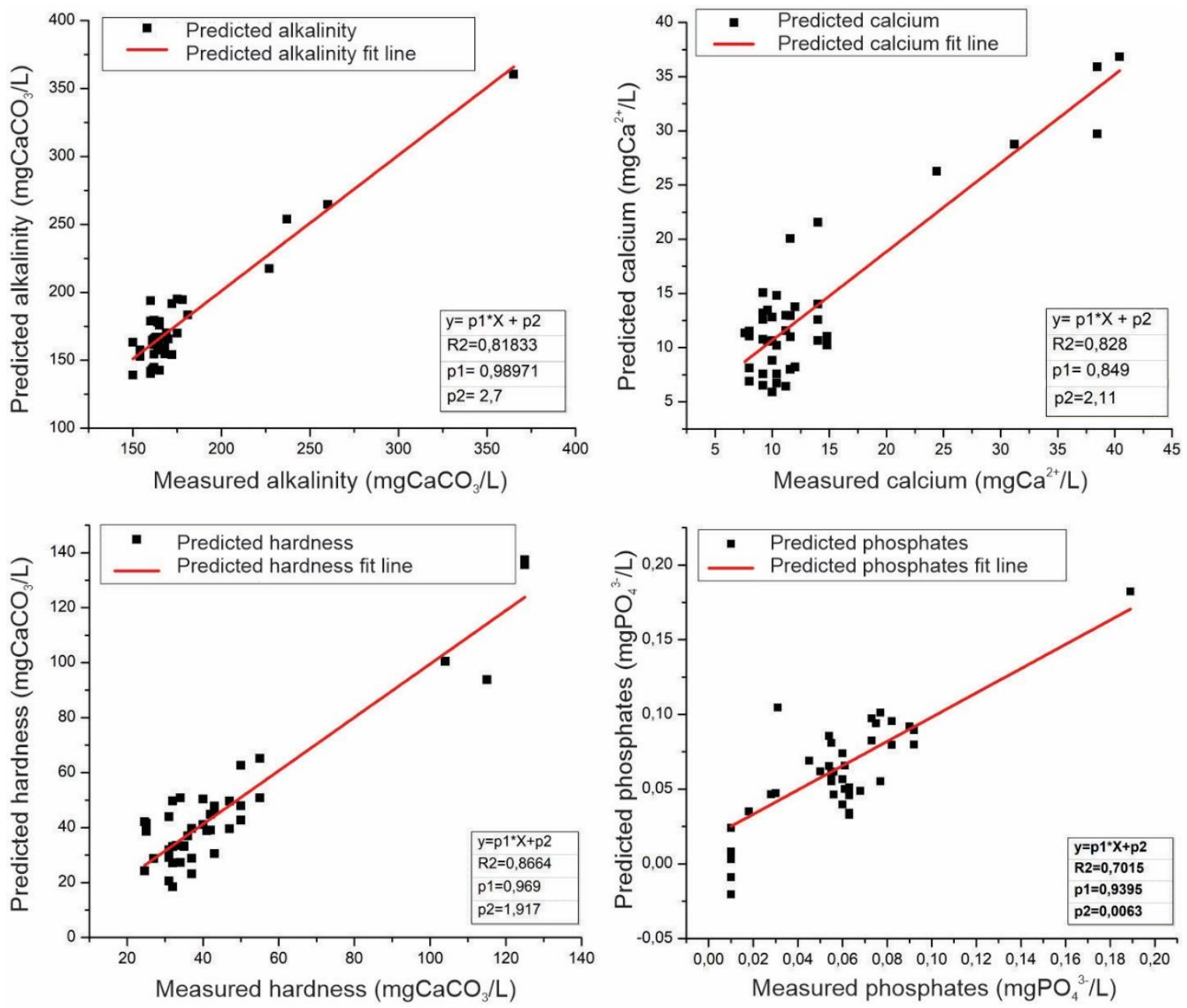

Figure 7. Regression models of physicochemical parameters (alkalinity, calcium, hardness and phosphates) generated by smart electronic tongue and traditional methods of chemical analysis. 
https://doi.org/10.5194/dwes-2021-12

Preprint. Discussion started: 14 January 2022

(c) Author(s) 2022. CC BY 4.0 License.

(c) (i)

275 It can be seen that the R2 (coefficient of determination) reached values of 0.701 in the case of phosphate, 0.818 for alkalinity and 0.828 and 0.866 for calcium and hardness respectively. Therefore, it can be considered that the smart electronic tongue presented good ability to predict the concentration of these substances.

In Figure 8 the graphs obtained for the physicochemical parameters, residual chlorine, chlorides, magnesium and sulphates are presented. In this case, linear correlation can be observed with R2 values of 0.315 for residual chlorine, 0.70 for chlorides, 0.788 for sulphate content and 0.825 for magnesium content. The R2 values obtained in the case of residual chlorine show low correlation, which may be due to the fact that residual chlorine is a not very stable parameter.
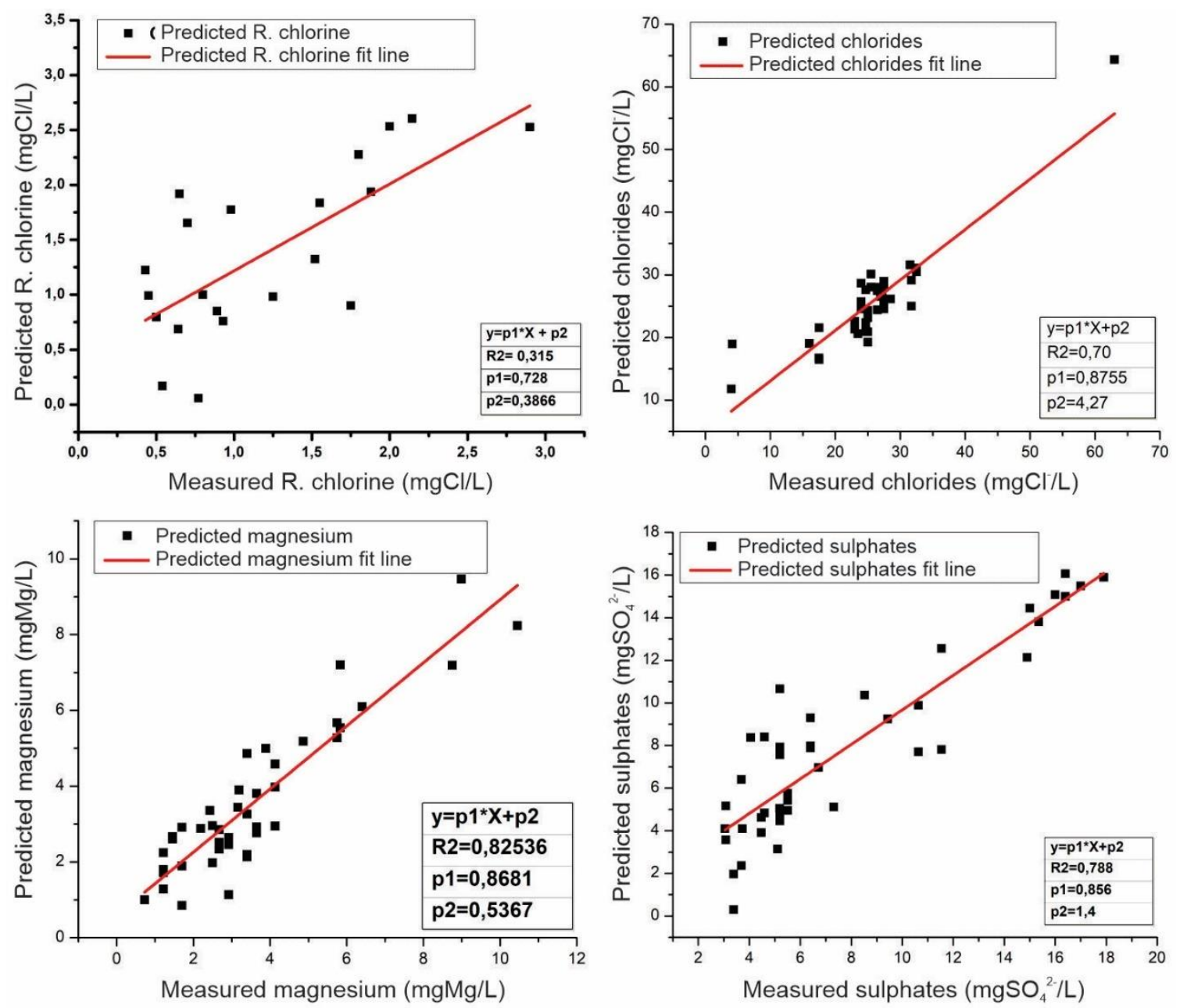

Figure 8. Regression models of physicochemical parameters (residual chlorine, chlorides, magnesium and sulphates) generated by smart electronic tongue and traditional methods of chemical analysis. 
https://doi.org/10.5194/dwes-2021-12

Preprint. Discussion started: 14 January 2022

(c) Author(s) 2022. CC BY 4.0 License.

(c) (i)

The instability of the residual chlorine in the water can be caused by the volatility of the chlorine, which is highly affected by light and high temperatures and although the samples were refrigerated in the sampling process, the city of Sincelejo is a city of an area tropical that registers an annual average temperature of $27^{\circ} \mathrm{C}$, which can affect both the traditional chemical analyzes carried out in the reference laboratory, as well as the measurements carried out with the smart electronic tongue. Otherwise, the correlation values obtained for chlorides, magnesium and sulphates, showed a high correlation between the two analytical approaches, which allows to establish a significant predictive capacity of the concentration of these substances by smart electronic tongue.

In general, of the eight physicochemical parameters studied, seven registered R2 values higher than 0.70, which can be considered values for highly correlated situations and only one parameter obtained a value lower than 0.49 , possibly due to the instability of the analyzed substance. It can be confirmed that the signals registered with the smart electronic tongue contain quantitative information, which can be analyzed through the application of regression models.

\section{Conclusions}

The results of the study allowed to conclude that the voltammetric signals registered by the sensor array of the smart electronic

300 tongue in samples of drinking water showed cross sensitivity, that is to say, each sensor in the array registered a different signal against one drinking water sample, also the signals of the recorded drinking water samples were different from each other, constituting this in a pattern or "fingerprint" of each sample analyzed. Each measurement took about 4 minutes to carried out, which represents a very reduced time when compared with the traditional methods of chemical analysis used in the physicochemical characterizations of water samples.

305 This behavior has allowed, through the application of artificial neural networks for principal components, to establish a discrimination between drinking water samples, a fact that reflects a good discrimination capacity of the smart electronic tongue sensor array. The results obtained with the analysis of the 22 samples and their replicas, showed an excellent discrimination capacity of the smart electronic tongue, with reproducible discrimination results.

Also, it could be shown that the smart electronic tongue provides quantitative information of some of the physicochemical

310 parameters of importance in the evaluation of the quality of drinking water. For this, the data were treated using regression models, with the aim of extracting quantitative information from the signals. Coefficient of determination values higher than 0.70 were established, which evidenced the excellent capacity of smart electronic tongue to provide information on substances of analytical interest that determine the quality of drinking water.

Finally, could be shown that smart electronic tongue allowed, from a single measurement, to perform qualitative and quasi315 quantitative analysis of the samples, which makes it a fast, efficient and economical analytical method. 
https://doi.org/10.5194/dwes-2021-12

Preprint. Discussion started: 14 January 2022

(c) Author(s) 2022. CC BY 4.0 License.

\section{(c) (1)}

\section{References}

Arrieta, A. A., Arrieta, P. L., and Mendoza, J. M.: Analysis of coffee adulterated with roasted corn and roasted soybean using voltammetric electronic tongue, Acta Sci.Pol. Technol. Aliment., 18(1), 35-41, https://doi.org/10.17306/J.AFS.2019.0619, 2019.

Arrieta, A. A., and Fuentes, O.: Portable electronic tongue for the analysis of raw milk based on PSoC (Programmable System on Chip) and Android technology, Ingeniare. Revista Chilena de Ingeniería, 24(3), 445-453, http://dx.doi.org/10.4067/S0718-33052016000300009, 2016.

Arrieta, A., Diaz, J., and Fuentes, O.: Optimization of the synthesis of polypyrrole for the elaboration of a network of sensors electrochemical (electronic tongue). Rev. Soc. Quím. Perú, 82(2), 152-161, http://www.scielo.org.pe/pdf/rsqp/v82n2/a06v82n2.pdf, 2016.

Arrieta, A., Fuentes, O., and Palencia, M.: Android and PSoC Technology Applied to Electronic Tongue Development, Research Journal of Applied Sciences, Engineering and Technology, 10(7), 782-788, DOI:10.19026/rjaset.10.2431, 2015.

Ataş, H. B., Kenar, A., and Taştekin, M.: An electronic tongue for simultaneous determination of $\mathrm{Ca} 2+, \mathrm{Mg} 2+, \mathrm{K}+$ and NH4+ in water samples by multivariate calibration methods, Talanta, 217, 1-12, https://doi.org/10.1016/j.talanta.2020.121110, 2020.

Aydemir, F. and Ebeoglu, M. A.: A QCM Sensor Array-Based Electronic Tongue with the Optimized Oscillator Circuit Using FPGA, IEEE Transactions on Instrumentation and Measurement, 67(2), 431-438, https://doi.org/10.1109/TIM.2017.2779318, 2018.

Belugina, R. B., Monakhova, Y. B., Rubtsova, E., Becht, A., Schollmayer, C., Holzgrabe, U., et al.: Distinguishing paracetamol formulations: Comparison of potentiometric "Electronic Tongue" with established analytical techniques, Journal of Pharmaceutical and Biomedical Analysis, 188, 1-7, https://doi.org/10.1016/j.jpba.2020.113457, 2020.

Dias, L. G., Alberto, Z., Veloso, A. C. A., and Peres, A. M.: Electronic tongue: a versatile tool for mineral and fruit-flavored waters recognition, Journal of Food Measurement and Characterization, 10(2), 264-273, https://doi.org/10.1007/s11694015-9303-y, 2015.

Khan, M., Khalilian, A., and Kang, S.: A High Sensitivity IDC-Electronic Tongue Using Dielectric/Sensing Membranes with Solvatochromic Dyes, Sensors, 16, 1-20, https://doi.org/10.3390/s16050668, 2016.

Kong, X., Hu, C., and Duan, Z.: Neural Networks for Principal Component Analysis. Springer. Principal Component Analysis Networks and Algorithms, Singapore. p. 47-73, 10.1007/978-981-10-2915-8, 2017.

Kovacs, Z., Szöllősi, D., Zaukuu, J. L. Z., Bodor, Z., Vitális, F., Aouadi, B., et al.: Factors Influencing the Long-Term Stability of Electronic Tongue and Application of Improved Drift Correction Methods, Biosensors, 10(7), 1-22, https://doi.org/10.3390/bios10070074, 2020. 
https://doi.org/10.5194/dwes-2021-12

Preprint. Discussion started: 14 January 2022

(c) Author(s) 2022. CC BY 4.0 License.

\section{(c) (i)}

Legin, E., Zadorozhnaya, O., Khaydukova, M., Kirsanov, D., Rybakin, V., Zagrebin, A., et al.: Rapid Evaluation of Integral Quality and Safety of Surface and Waste Waters by a Multisensor System (Electronic Tongue), Sensors, 19(9), 1-15, https://doi.org/10.3390/s19092019, 2019.

Li, S., Li, K., Li, X., and Chen, Z.: A colorimetric electronic tongue for rapid discrimination of antioxidants based on the oxidation etching of nano-triangular silver by metal ions, ACS Applied Materials \& Interfaces, 11, 37371-37378, https://doi.org/10.1021/acsami.9b14522, 2019.

Marx, Í, M. G., Rodrigues, N., Dias, L. G., Veloso, A. C. A., Pereira, J. A., Drunkler, D. A., et al.: Quantification of table olives' acid, bitter and salty tastes using potentiometric electronic tongue fingerprints, LWT - Food Science and Technology, 79, 394-401, https://doi.org/10.1016/j.1wt.2017.01.060, 2017.

National Institute of Health, Subdirectorate of the National Laboratory Network, Laboratory Surveillance Program of Water Quality for Human Consumption, Bogota National Institute of Health. https://www.ins.gov.co/Direcciones/ RedesSaludPublica/DocumentosdeInteresSRNL/Manual_toma_envio_muestras_INS-2019.pdf, 2019.

Rice, E.W., Baird, R. B., and Eaton, A. D.: Standard Methods for the Examination of Water and Wastewater. 23rd Edition. American Public Health Association, American Water Works Association, Water Environmental Federation. New York, 2017.

Richardson, S. D., and Ternes, T. A.: Water Analysis: Emerging Contaminants and Current Issues, Analytical Chemistry, 90 (1), 398-428, https://doi.org/10.1021/acs.analchem.7b04577, 2017.

Sehra, G., Cole, M., and Gardner, J. W.: Miniature taste sensing system based on dual SH-SAW sensor device: an electronic tongue, Sensors and Actuators B: Chemical, 103(1-2), 233-239, https://doi.org/10.1016/j.snb.2004.04.055, 2004.

Sincelejo mayorship: Sincelejo land use plan, agreement 177 of 2017, Urban Regulation: Sincelejo Mayor's Office: https://www.alcaldiadesincelejo.gov.co/Transparencia/PlaneacionGestionyControl/Acuerdo\%20N\%C2\%B0\%20177\%20 de\%202017.PDF.

Sipos, L., Kovács, Z., Sági-Kiss, V., Csiki, T., Kókai, Z., Fekete, A., et al.: Discrimination of mineral waters by electronic tongue, sensory evaluation and chemical analysis, Food Chemistry, 135(4), 2947-2953, https://doi.org/10.1016/j.foodchem.2012.06.021, 2012.

Titova, T., and Nachev, V.: "Electronic tongue" in the Food Industry, Food Science and Applied Biotechnology, 3(1), 71-76, https://doi.org/10.30721/fsab2020.v3.i1.74, 2020.

Vlasov, Y., Legin, A., Rudnitskaya, A., Di Natale, C., and D'Amico, A.: Nonspecific sensor arrays ("electronic tongue") for chemical analysis of liquids (IUPAC Technical Report), Pure and Applied Chemistry, 77(11), 1965-1983, https://doi.org/10.1351/pac200577111965, 2005.

Winquist, F., Olsson, J., and Eriksson, M.: Multicomponent analysis of drinking water by a voltammetric electronic tongue, Analytica Chimica Acta, 683(2), 192-197, https://doi.org/10.1016/j.aca.2010.10.027, 2011. 